\title{
Newborn screening for X-linked adrenoleukodystrophy: evidence summary and advisory committee recommendation
}

\author{
Alex R. Kemper, MD, MPH${ }^{1}$, Jeffrey Brosco, MD, $\mathrm{PhD}^{2}$, Anne Marie Comeau, $\mathrm{PhD}^{3}$, \\ Nancy S. Green, MD ${ }^{4}$, Scott D. Grosse, $\mathrm{PhD}^{5}$, Elizabeth Jones, $\mathrm{MPH}^{6}$, Jennifer M. Kwon, MD , \\ Wendy K.K. Lam, PhD' ${ }^{1}$, Jelili Ojodu, $\mathrm{MPH}^{6}$, Lisa A. Prosser, $\mathrm{PhD}^{8}$ and Susan Tanksley, $\mathrm{PhD}^{9}$
}

The secretary of the US Department of Health and Human Services in February 2016 recommended that X-linked adrenoleukodystrophy (X-ALD) be added to the recommended uniform screening panel for state newborn screening programs. This decision was informed by data presented on the accuracy of screening from New York, the only state that currently offers X-ALD newborn screening, and published and unpublished data showing health benefits of earlier treatment (hematopoietic stem cell transplantation and adrenal hormone replacement therapy) for the childhood cerebral form of X-ALD. X-ALD newborn screening also identifies individuals with later-onset disease, but poor genotype-phenotype correlation makes predicting health outcomes difficult and might increase the risk of unnecessary treatment. Few data are available regarding the harms of screening and presymptomatic identification. Significant challenges exist for implementing comprehensive X-ALD newborn screening, including incorporation of the test, coordinating follow-up diagnostic and treatment care, and coordination of extended family testing after case identification.

Genet Med advance online publication 23 June 2016

Key Words: adrenoleukodystrophy; dried blood spot testing public health; evidence-based practice; neonatal screening

\section{INTRODUCTION}

Nearly all of the approximately 4 million newborns in the United States undergo screening within the first few days of life, primarily through evaluation of dried blood spots for a wide variety of conditions, with the goal of improving health outcomes through early detection. Each state determines which conditions are included in its screening program. To assist state newborn screening programs, the secretary of the US Department of Health and Human Services maintains a federally recommended uniform screening panel (RUSP), a listing of those conditions for which there is thought to be sufficient evidence that newborn screening leads to improved health outcomes compared to usual case detection and that these improved health outcomes outweigh the harms of screening. The secretary's decision to add conditions to the RUSP is based, in part, on the recommendations from the Advisory Committee on Heritable Disorders in Newborns and Children (hereafter the "Advisory Committee").

The Advisory Committee develops recommendations informed by a review of the evidence presented by the Condition Review Workgroup. This workgroup is external to the Advisory Committee and is not involved with developing or voting on the recommendations. Instead, the Condition Review
Workgroup provides three components: a systematic evidence review of published reports and unpublished data; evaluation of the expected population impact of implementation of screening, based on modeling; and an assessment of feasibility and readiness of state newborn screening programs to implement newborn screening for the condition under consideration.

In September 2012, X-linked adrenoleukodystrophy (X-ALD) was nominated to be considered as an addition to the RUSP. At that time, the Advisory Committee did not find sufficient evidence to add X-ALD to the RUSP; therefore, a full evidence review was not conducted. However, by September 2014, the Advisory Committee determined that the body of evidence had increased, based on pilot work that had begun in New York, and therefore moved forward with its evaluation and recommendation process. This report by the Condition Review Workgroup summarizes the Advisory Committee's recommendation regarding newborn screening for X-ALD and the evidence provided to the Advisory Committee to inform its decision. The full report provided by the Condition Review Workgroup and the Advisory Committee's letter to the secretary of Health and Human Services is available at http://www.hrsa.gov/advisorycommittees/mchbadvisory/heritabledisorders/nominatecondition/workgroup.html. The online

\footnotetext{
${ }^{1}$ Duke Clinical Research Institute, Duke University, Durham, North Carolina, USA; ${ }^{2}$ Department of Pediatrics, University of Miami, Coral Gables, Florida, USA; ${ }^{3}$ Department of Pediatrics, University of Massachusetts Medical School, Jamaica Plain, Massachusetts, USA; ${ }^{4}$ Department of Pediatrics, Columbia University Medical Center, New York, New York, USA; ${ }^{5}$ ational Center on Birth Defects and Developmental Disabilities, Centers for Disease Control and Prevention, Atlanta, Georgia, USA; ${ }^{6}$ Association of Public Health Laboratories, Silver Spring, Maryland, USA; ${ }^{7}$ Department of Neurology, University of Rochester Medical Center, Rochester, New York, USA; ${ }^{8}$ Child Health Evaluation and Research Unit, Department of Pediatrics, University of Michigan, Ann Arbor, Michigan, USA; ${ }^{\top}$ Texas Department of State Health Services, Austin, Texas, USA. Correspondence: Alex R. Kemper (alex.kemper@duke.edu)
} 
report contains a complete description of methods and findings related to published and unpublished data.

\section{ADVISORY COMMITTEE RECOMMENDATION}

The Advisory Committee recommended in September 2015 that X-ALD be added to the RUSP. The Advisory Committee also recommended that federal funds be made available to state newborn screening programs to assist with implementation. On 16 February 2016, the secretary agreed with the recommendation to add X-ALD to the RUSP. Although no new funding was made available for implementation, the secretary requested that federal agencies consider how to support state programs using existing resources.

\section{APPROACH TO EVIDENCE REVIEW AND SYNTHESIS}

The evidence review was developed to answer a series of key questions necessary to inform the Advisory Committee (see Table 1). A technical expert panel that included six experts in X-ALD screening, diagnosis, and treatment was convened to critically review the work plan and to provide guidance on sources of relevant evidence. These individuals were selected because they had expertise in screening, diagnosis, and treatment based on review of publications in the field. The technical expert panel did not participate in the development of Advisory Committee recommendations.

The primary source of evidence was based on a systematic review of published peer-reviewed publications using several databases (MEDLINE, EMBASE, and CINAHL) from inception through July 2015. Articles based on human subject data published in English and including any study design were selected. The Condition Review Workgroup recognizes that there are new therapies in development (e.g., gene therapy) that were not included in the review because of the inability to assess benefits or harms. We also did not evaluate treatment with Lorenzo's oil because of questions about its effectiveness and the emergence of hematopoietic stem cell transplantation (HSCT) as a treatment option. Overall, 1,314 articles were initially included and 172 had relevant evidence for the key questions. Findings were supplemented with two data sets describing treatment outcomes: a single-center registry maintained by investigators at the Massachusetts General Hospital for Children and covering treatment for 30 patients from 2006 to 2015 , and a multisite international registry maintained by BlueBird Bio (Cambridge, MA) of 59 patients with X-ALD treated with HSCT in 2001 or later. There was no overlap in the subjects across the two registries. The Condition Review Workgroup was given direct access to the data to create summary statistics for the Advisory Committee. However, because the owners of the databases plan for future separate independent publications, the Condition Review Workgroup was required to embargo detailed findings from the public. Although the registry data appeared complete and valid, the Condition Review Workgroup emphasized the small number of subjects and the potential for selection bias.

\section{X-ALD overview \\ SUMMARY OF THE EVIDENCE}

X-ALD (OMIM 300100) is a peroxisomal disorder caused by mutations in the $\mathrm{ABCD} 1$ gene $^{1}$ and is estimated to affect approximately 1 in 42,000 males. ${ }^{2}$ Additionally, 1 in 28,000 females are estimated to be heterozygous for an ABCD1 mutation. ${ }^{2}$ The disorder has a broad phenotype and there is no genotypephenotype correlation, even within families. Data extrapolated from reported incident cases suggest that the most severe form, childhood cerebral ALD (CCALD), affects between 31 and 57\% of hemizygous males. ${ }^{1,3-6}$ CCALD typically presents between 2 and 10 years of age and is associated with rapid neurologic decline; without treatment, death or severe disability typically occurs within approximately 3 years. ${ }^{78}$ For boys who have been determined to have CCALD, treatment is HSCT administered at an early stage of progressive brain involvement. Because most boys with X-ALD will not develop CCALD, and because of the risks associated with HSCT, HSCT is reserved for those with a clear diagnosis of CCALD in its early stages. ${ }^{5,7-9}$

Most males with CCALD will also develop adrenal insufficiency. ${ }^{10,11}$ If unrecognized, delayed treatment of adrenal insufficiency can lead to death from even minor illnesses. Little is known about how often signs or symptoms of adrenal insufficiency lead to the diagnosis of X-ALD or about the timing of clinical recognition of adrenal insufficiency relative to the

Table 1 Summary of key questions used in the development of the systematic evidence review

General topic
Natural history
Short-term follow-up and
diagnostic confirmation

Treatment and long-term follow-up

\section{Key questions}

What are the natural history and epidemiology of X-ALD? What are the estimated incidence rates for the associated phenotypes and the typical course of disease? What factors predict morbidity or mortality?

What are the direct and indirect evidence that newborn screening for X-ALD leads to improved health outcomes compared to usual clinical care? What is the analytic validity or clinical validity of the newborn screening approaches used to detect X-ALD? What diagnostic methods are available to confirm or identify the phenotypes? What methods are available to predict or inform the age of onset or disease severity? What harms to the individual or family are associated with newborn screening for X-ALD?

What are the standard treatments for X-ALD and evidence for their effectiveness? Do follow-up protocols that do not require immediate initiation of treatment exist for the management of X-ALD? What is known about the effectiveness of follow-up protocols in modifying health outcomes? What are the harms associated with treatments for X-ALD in early childhood for symptomatic or presymptomatic patients? How does this vary by phenotype? What is the impact of newborn screening on the public health of the population or on the public health system? What is the feasibility of population-based screening for X-ALD in the United States? 
development of cerebral involvement. HSCT treatment for CCALD does not treat adrenal hormone insufficiency; therefore, lifelong hormone replacement therapy is required for all $\mathrm{X}$-ALD patients with adrenal insufficiency.

Affected males with or without CCALD may develop progressive spastic paraparesis, sensory ataxia, and other peripheral nerve and spinal nerve involvement, collectively referred to as adrenomyeloneuropathy (AMN). Relatively little is known about the natural history of $\mathrm{AMN}$. Although the literature indicates that the typical age of AMN onset is after age 30, the neurological symptoms may begin to manifest in later childhood or adolescence. Patients with AMN can develop adrenal insufficiency years or even decades before the disease is recognized based on the onset of neurological symptoms. The course of AMN is highly variable; within a mean of 13 years from recognized onset in adults, the rate of death or severe disability is reported to be $12 \%{ }^{5}$ Although AMN is not amenable to HSCT, identification and treatment of adrenal insufficiency in these patients can be lifesaving. No studies that characterized the morbidity associated with delayed recognition of adrenal insufficiency in any type of X-ALD were identified.

Heterozygous females can develop symptoms, usually of AMN, in mid- to late adulthood. ${ }^{12}$ Although potentially serious, the impact of heterozygous females manifesting some form of $\mathrm{X}$-ALD was not explicitly considered by the Condition Review Workgroup because of the adult onset of symptoms.

\section{X-ALD newborn screening and diagnostic strategies}

$\mathrm{X}$-ALD is characterized by persistently elevated serum levels of particular very-long-chain fatty acids, and the diagnosis is confirmed by identifying ABCD1 gene mutations. Although ABCD1 mutations cause X-ALD, the presence of mutations does not predict disease course, even within families. To identify the subset of males who will develop CCALD and who could potentially be treated with HSCT, all males with ABCD1 mutations require ongoing clinical assessment, serial magnetic resonance imaging (MRI) studies of the brain, and assessment of adrenocortical function to guide treatment. ${ }^{7-9,13}$

Newborn screening for X-ALD is based on the elevation of a lysophosphatidylcholine derivative of a very-long-chain fatty acid marker in dried blood spots, abbreviated C26:0-LPC. New York uses a two-tiered screening approach based on tandemmass spectrometry (MS/MS). ${ }^{14}$ The first tier uses flow-injection analysis MS/MS to measure C26:0-LPC. The second tier test is used for samples with elevated C26:0-LPC and relies on MS/ MS coupled with high-performance liquid chromatography to measure C26:0-LPC in the same dried blood spot. In New York, these tests are currently multiplexed with the newborn screening tests for Krabbe disease and Pompe disease. Newborns with elevated levels of C26:0-LPC on the second tier are referred for confirmatory testing. To assist in the evaluation process, the New York Newborn Screening Program also routinely performs Sanger sequencing to determine if there are mutations in the ABCD1 gene. The sequencing is generally completed prior to the referral. In addition to screening for X-ALD, this approach can also identify other peroxisomal disorders (e.g., Zellweger syndrome) often associated with death in early infancy and for which there is no known treatment.

Over the 18-month period ending in July 2015, approximately 365,000 newborns were screened for X-ALD in New York. Approximately 1.8\% required the second tier test and 33 newborns were referred for confirmatory testing. In this period, 13 males had ABCD1 mutations identified by the New York newborn screening laboratory. Newborn screening identified 13 heterozygous females and one male with Klinefelter syndrome, four with Zellweger syndrome, one case of AicardiGoutieres syndrome, and one critically ill newborn not further characterized. Clinical experts anticipate that the infant with Klinefelter syndrome will have a course similar to that of the females who are heterozygous for X-ALD. It is not known why an infant with Aicardi-Goutieres syndrome was identified through newborn screening, because it is not typically associated with elevated very-long-chain fatty acids. The identification of these nontargeted conditions illustrates the general experience of newborn screening, which often picks up conditions other than those specifically targeted.

In New York, testing is offered to members of the extended family after diagnosis of X-ALD or identification of heterozygote females through newborn screening. No data were available regarding the impact of carrier testing on families or the uptake or outcomes of extended family testing for X-ALD.

\section{Surveillance and treatment of X-ALD}

At the time of the evidence review, it was recommended that asymptomatic males identified through newborn screening in New York have their adrenal function quantitatively assessed every 6 months and brain MRI annually from 6 months to 30 months of age, then every 6 months from 3 to 10 years of age, and then annually again until 18 years of age. ${ }^{14}$ More recently, the New York newborn screening program has recommended not obtaining the first MRI until 12 months of age, and then annually until 3 years of age, followed by every 6 months to 10 years of age, and then annually (J.M.K., personal communication).

In addition to supportive care, the current treatments include cortisol for individuals with adrenal insufficiency and HSCT for individuals with CCALD at the early stages of progressive brain involvement. HSCT has been reported to slow or even arrest the progression of leukodystrophy, ${ }^{15-26}$ but it does not modify the course of adrenal insufficiency or other types of myelopathy and neuropathy.

A limitation of the evidence reviewed was that no published study directly compared treatment outcomes for individuals detected presymptomatically (e.g., through newborn screening or family history) with those diagnosed symptomatically. Indirect evidence suggests that earlier age of treatment with HSCT is associated with better outcomes. For example, more advanced disease, as indicated by increased white matter pathology found on brain MRI, is associated with worse outcomes following HSCT. ${ }^{16,22,23}$ Unpublished data in small 
numbers of subjects suggest that detection through family testing (i.e., presymptomatic identification of a young child who had a relative previously identified with X-ALD), compared with clinical detection resulting from testing based on signs or symptoms, is associated with less cerebral disease at the initiation of HSCT and also with longer survival and improved neurocognitive outcomes.

No data regarding the specific benefit of presymptomatic detection of adrenal insufficiency were identified. Cortisol for adrenal hormone insufficiency has been studied in many other conditions and is recognized as efficacious. However, little is known about adrenal insufficiency specifically associated with X-ALD, including long-term prognosis, adherence to cortisol treatment, or the clinical consequences of incomplete adherence.

\section{Potential harms of X-ALD newborn screening}

An important potential harm of X-ALD newborn screening is direct clinical harm related to treatment following early diagnosis. No recent data are available regarding the mortality risk associated with HSCT for X-ALD. However, among studies reporting transplant follow-up at 1 year or later, the overall 1-year risk of mortality following HSCT appears to be approximately $15 \%$ or less. ${ }^{15,19,21-23,27-29}$ The mortality rate is a function of several factors, including the health of the child at the time of HSCT, the type of transplantation performed, the degree of match between donor and recipient, and the treatment regimen used by those performing the HSCT. Due to lack of data, the Condition Review Workgroup was unable to assess the potential availability of well-matched donors if newborn screening were adopted. Insufficient evidence was available regarding the availability of treatment centers to offer HSCT, specifically for $\mathrm{X}$-ALD, given the concomitant needs of having on-site clinical expertise in the management of X-ALD-related comorbidities and complications. In general, HSCT has a significant risk of mortality and morbidity. For example, a case series of 51 infants treated with HSCT between 1992 and 2010 with long-term follow-up found an overall survival rate of $70 \%$ after a median follow-up of 8.9 years, and 30\% had acute graft-versus-host disease..$^{30}$ Although such harms might occur in children with $\mathrm{X}$-ALD regardless of how the condition was detected, some children who would not have undergone transplantation in the absence of screening might undergo HSCT. There is also the potential harm that individuals could receive inappropriate or ineffective treatment.

As with all newborn screening programs, there is a risk of false-positive results in an infant without the condition or a positive result that leads to overdiagnosis. For example, female heterozygotes often do not develop symptoms for decades. Although knowing the carrier status might inform reproductive decision making, some families might not want the information at birth. Individuals and families can become anxious because of the inability to predict phenotypic expression of the condition, which for some males might not be for decades. ${ }^{31}$ Because knowledge about the management of X-ALD detected presymptomatically is still developing, there is a risk that an individual could be treated with HSCT sooner than is clinically indicated and thus could face the risks of HSCT at a younger age or being matched with an imperfectly matched donor when a better match might become available later. Delaying the first MRI to 1 year of age can help decrease the risk of overtreatment. Despite the broad range of potential harms, no study was included that specifically evaluated these harms for X-ALD newborn screening.

Projected benefits and harms of population-level screening A limitation of the evidence reviewed was that no published study directly compared treatment outcomes for individuals detected presymptomatically (e.g., through newborn screening or family history) with those diagnosed after the development of signs or symptoms. Modeling was used to summarize the expected population-level impact of implementing newborn screening. Model inputs included published and unpublished data supplemented by expert input regarding the birth incidence of X-ALD, the natural history of X-ALD in unscreened populations, the accuracy of screening, and the impact of treatment stratified by the degree of cerebral involvement. It was necessary to modify the model inputs because of uncertainty regarding the impact of newborn screening on the observed epidemiology of X-ALD. Because of the limited available data, the analysis only considered outcomes through 15 years of age. Long-term outcome data came directly from the two unpublished data sets identified by the Condition Review Workgroup. Furthermore, it was not possible to separately consider the impact of HSCT and cortisol treatment. Based on this analysis, screening all 4 million newborns in the United States each year for X-ALD could lead to the identification of 143 (range: 64-211) newborns with a mutation in the ABCD1 gene compared to 92 (range: 64-132) cases that would be detected through usual clinical care. Although the total number of cases of CCALD expected to be identified through newborn screening (46; range: $32-68$ ) would be similar to the number detected through clinical care (46; range: 32-66), earlier detection could allow HSCT to be performed earlier in the disease course, thus potentially improving health outcomes. Based on incomplete unpublished data, by 15 years of age, no deaths would be expected in the group identified by screening (range: $0-21)$ compared to 18 deaths in the group with only clinical detection (range: 7-44). In those identified through newborn screening compared to those identified through clinical care, fewer cases of severe outcomes (e.g., patients who are nonambulatory and noncommunicative) would also be expected for survivors. These estimates are preliminary and relied on expert opinion. These results are likely to change as more data become available.

\section{Newborn screening program implementation}

New York currently screens for X-ALD according to the legislative mandate. Three other states (California, Connecticut, and New Jersey) also have a legislative mandate for X-ALD 
newborn screening, but as of the time of this writing they had not yet begun screening. A survey of state newborn screening programs found that most believed that the technology to begin screening for X-ALD could be in place within 1 to 3 years after funds were allocated. Newborn screening programs face the challenge of access to sufficient funds to expand newborn screening and the challenge of adding other recommended conditions to the panel (e.g., severe combined immunodeficiency).

\section{ADDITIONAL CLINICAL CONSIDERATIONS}

Several important considerations relate to follow-up after case identification. Clinical referral networks need to be established not only to provide diagnostic and treatment services but also to coordinate follow-up for individuals with presymptomatic $\mathrm{X}$-ALD. These clinical referral networks require expertise in medical genetics, neurology, and endocrinology, and in the provision of HSCT for CCALD. It is important to recognize that HSCT is only currently recommended for those who meet criteria for CCALD at an early stage of progressive leukodystrophy. Therefore, each clinic providing follow-up care will have to be available to carefully monitor those identified by newborn screening and to be able to appropriately refer for HSCT, either within its network or elsewhere, when the criteria are met. The referral networks also have to be prepared to offer extended family testing after case identification through newborn screening. The Advisory Committee, based on its standard procedure at the time of this review, did not explicitly consider public health costs related to implementation of X-ALD.

The benefit of early detection compared to usual case detection was based primarily on unpublished data, including an analysis of proprietary registry data. Evaluating outcomes of screening and different approaches to diagnostic follow-up and treatment is critical to improving the care received by individuals identified with X-ALD through screening. Well-designed registry data could support quality improvement activities, revision of clinical care guidelines, and monitoring of treatments and long-term outcomes, including neurologic impairment and development of adrenal insufficiency. Development of a nonproprietary multisite registry would allow ongoing assessment not only of the benefits of X-ALD newborn screening but also of the harms, which have not been the focus of previous work in the field. As with all rare disorders, pooling data is critical to refine approaches to diagnosis and treatment.

The Condition Review Workgroup identified concerns regarding the accessibility of clinical experts with experience in diagnosing and treating X-ALD needed to support populationbased screening of X-ALD. Access to these specialists is limited by their availability, geographic location, and aspects related to financing (e.g., the challenge of Medicaid payment across state lines). Lack of clinical expertise or limited access to care could lead to harm for those with a positive newborn screen, such as provision of inappropriate care.

The Condition Review Workgroup evaluated screening both males and females as is typically done by state newborn screening programs compared to screening males only. However, female heterozygotes do not develop significant symptoms in childhood. The routine reporting of results to families regarding children with genetic markers for adult-onset disease is controversial, ${ }^{32}$ but it can be a strategy for identifying other cases through extended family testing.

Future work is needed to assess the availability of suitable matches for HSCT. Review of evidence was not able to provide information about the proportion of children who would have a match, but it noted that the use of banked umbilical cord blood could extend the pool of potential matches because umbilical cord blood does not require the same degree of matching as donor marrow.

\section{CONCLUSIONS}

Data from New York suggest that X-ALD can be reliably identified through newborn screening. Not all cases of X-ALD will require treatment, but all cases of $\mathrm{X}-\mathrm{ALD}$ require monitoring for a period of years to determine if treatment is indicated. Monitoring will include periodic brain MRI studies and testing of adrenal function, and it requires close supervision by specialists with experience in X-ALD. Although it is too early to assess whether screening in New York has led to improvements in health outcomes, unpublished evidence from other sources suggests that earlier detection of CCALD can improve survival and neurologic function through HSCT. In addition, screening could identify individuals who will develop adrenal insufficiency, which can be asymptomatic and can be treated with cortisol. Challenges include the poor genotype-phenotype correlation and the need to develop an infrastructure for both screening and follow-up care. Harms of screening include falsepositive results, overdiagnosis, and the risks associated with HSCT performed earlier than needed. High-quality registry data for X-ALD could improve knowledge about treatments and outcomes. Such a registry could also be helpful in evaluating the impact of future developments (e.g., improvements in phenotype prediction, gene therapy). The Condition Review Workgroup did not model the costs associated with X-ALD screening. In the future, the Condition Review Workgroup will provide such information as a component of the evidence review when data can be reliably obtained.

\section{ACKNOWLEDGMENTS}

This project was supported by the Health Resources and Services Administration (HRSA) of the US Department of Health and Human Services (HHS) under contract number HHSH250201400007C, "Evidence-based Reviews for the Discretionary Advisory Committee on Heritable Disorders in Newborns and Children."

The views expressed herein are solely those of the individual authors and do not necessarily reflect the views of the secretary of the US Department of Health and Human Services, the Centers for Disease Control and Prevention, or the Association of Public Health Laboratories. This information or content and conclusions are those of the authors and should not be construed as the official position or policy of, nor should any endorsements be inferred by, HRSA, HHS, or the US Government. 


\section{DISCLOSURE}

The authors declare no conflict of interest.

\section{REFERENCES}

1. Kirk EP, Fletcher JM, Sharp P, Carey B, Poulos A. X-linked adrenoleukodystrophy: the Australasian experience. Am J Med Genet 1998;76:420-423.

2. Bezman L, Moser AB, Raymond GV, et al. Adrenoleukodystrophy: incidence, new mutation rate, and results of extended family screening. Ann Neurol 2001:49:512-517.

3. Ruiz M, Coll MJ, Pàmpols T, Girós M. X-linked adrenoleukodystrophy: phenotype distribution and expression of ALDP in Spanish kindreds. Am J Med Genet 1998;76:424-427.

4. van Geel BM, Assies J, Weverling GJ, Barth PG. Predominance of the adrenomyeloneuropathy phenotype of X-linked adrenoleukodystrophy in The Netherlands: a survey of 30 kindreds. Neurology 1994;44:2343-2346.

5. Moser HW, Moser AB, Smith KD, et al. Adrenoleukodystrophy: phenotypic variability and implications for therapy. J Inherit Metab Dis 1992;15: 645-664.

6. Takemoto $Y$, Suzuki $Y$, Tamakoshi A, et al. Epidemiology of $X$-linked adrenoleukodystrophy in Japan. J Hum Genet 2002;47:590-593.

7. Moser HW, Mahmood A, Raymond GV. X-linked adrenoleukodystrophy. Nat Clin Pract Neurol 2007;3:140-151.

8. van Geel BM, Assies J, Wanders RJ, Barth PG. X linked adrenoleukodystrophy: clinical presentation, diagnosis, and therapy. J Neurol Neurosurg Psychiatry 1997:63:4-14.

9. Engelen $\mathrm{M}, \mathrm{Kemp} \mathrm{S}$, Poll-The BT. X-linked adrenoleukodystrophy: pathogenesis and treatment. Curr Neurol Neurosci Rep 2014;14:486.

10. Dubey P, Raymond GV, Moser AB, Kharkar S, Bezman L, Moser HW. Adrenal insufficiency in asymptomatic adrenoleukodystrophy patients identified by very long-chain fatty acid screening. J Pediatr 2005;146:528-532.

11. Korenke GC, Roth C, Krasemann E, Hüfner M, Hunneman DH, Hanefeld F. Variability of endocrinological dysfunction in 55 patients with X-linked adrenoleucodystrophy: clinical, laboratory and genetic findings. Eur J Endocrinol 1997; 137:40-47.

12. Engelen $M$, Barbier $M$, Dijkstra IM, et al. X-linked adrenoleukodystrophy in women: a cross-sectional cohort study. Brain 2014;137(Pt 3):693-706.

13. Engelen $M$, Kemp S, de Visser M, et al. X-linked adrenoleukodystrophy (X-ALD): clinical presentation and guidelines for diagnosis, follow-up and management. OrphanetJ Rare Dis 2012;7:51.

14. Vogel BH, Bradley SE, Adams DJ, et al. Newborn screening for X-linked adrenoleukodystrophy in New York State: diagnostic protocol, surveillance protocol and treatment guidelines. Mol Genet Metab 2015;114:599-603.

15. Mitchell R, Nivison-Smith I, Anazodo A, et al. Outcomes of haematopoietic stem cell transplantation for inherited metabolic disorders: a report from the Australian and New Zealand Children's Haematology Oncology Group and the Australasian Bone Marrow Transplant Recipient Registry. Pediatr Transplant 2013;17:582-588.

16. Miller WP, Rothman SM, Nascene D, et al. Outcomes after allogeneic hematopoietic cell transplantation for childhood cerebral adrenoleukodystrophy: the largest single-institution cohort report. Blood 2011;118:1971-1978.
17. Polgreen LE, Chahla S, Miller W, et al. Early diagnosis of cerebral X-linked adrenoleukodystrophy in boys with Addison's disease improves survival and neurological outcomes. Eur J Pediatr 2011;170:1049-1054.

18. Okamura K, Watanabe T, Onishi T, et al. Successful allogeneic unrelated bone marrow transplantation using reduced-intensity conditioning for the treatment of X-linked adrenoleukodystrophy in a one-yr-old boy. Pediatr Transplant 2009;13:130-133.

19. Mahmood A, Raymond GV, Dubey P, Peters C, Moser HW. Survival analysis of haematopoietic cell transplantation for childhood cerebral X-linked adrenoleukodystrophy: a comparison study. Lancet Neurol 2007;6:687-692.

20. Beam D, Poe MD, Provenzale JM, et al. Outcomes of unrelated umbilical cord blood transplantation for X-linked adrenoleukodystrophy. Biol Blood Marrow Transplant 2007;13:665-674.

21. Resnick IB, Abdul Hai A, Shapira MY, et al. Treatment of X-linked childhood cerebral adrenoleukodystrophy by the use of an allogeneic stem cell transplantation with reduced intensity conditioning regimen. Clin Transplant 2005; 19:840-847.

22. Peters C, Charnas LR, Tan Y, et al. Cerebral X-linked adrenoleukodystrophy: the international hematopoietic cell transplantation experience from 1982 to 1999. Blood 2004;104:881-888.

23. Baumann M, Korenke GC, Weddige-Diedrichs A, et al. Haematopoietic stem cell transplantation in 12 patients with cerebral X-linked adrenoleukodystrophy. Eur J Pediatr 2003;162:6-14.

24. Shapiro E, Krivit W, Lockman L, et al. Long-term effect of bone-marrow transplantation for childhood-onset cerebral X-linked adrenoleukodystrophy. Lancet 2000;356:713-718.

25. Shapiro EG, Lockman LA, Balthazor M, Krivit W. Neuropsychological outcomes of several storage diseases with and without bone marrow transplantation. J Inherit Metab Dis 1995;18:413-429.

26. Loes DJ, Stillman AE, Hite $S$, et al. Childhood cerebral form of adrenoleukodystrophy: short-term effect of bone marrow transplantation on brain MR observations. AJNR Am J Neuroradio/ 1994;15:1767-1771.

27. Gassas A, Raiman J, White L, Schechter T, Clarke J, Doyle J. Long-term adaptive functioning outcomes of children with inherited metabolic and genetic diseases treated with hematopoietic stem cell transplantation in a single large pediatric center: parents' perspective. J Pediatr Hematol Oncol 2011;33:216-220.

28. McKinney AM, Nascene D, Miller WP, et al. Childhood cerebral X-linked adrenoleukodystrophy: diffusion tensor imaging measurements for prediction of clinical outcome after hematopoietic stem cell transplantation. AJNR Am J Neuroradiol 2013;34:641-649.

29. Miller WP, Rothman SM, Nascene D, et al. Outcomes after allogeneic hematopoietic stem cell transplantation for childhood cerebral adrenoleukodystrophy: the largest single-institution cohort report. Blood 2010:116: 1971-1978:

30. Gassas A, Ashraf K, Zaidman I, et al. Hematopoietic stem cell transplantation in infants. Pediatr Blood Cancer 2015;62:517-521.

31. Timmermans $S$, Buchbinder M. Patients-in-waiting: Living between sickness and health in the genomics era. J Health Soc Behav 2010;51:408-423.

32. American Academy of Pediatrics Committee on Bioethics, Committee on Genetics, and the American College of Medical Genetics and Genomics Social Ethical and Legal Issues Committee. Ethical and Policy Issues in Genetic Testing and Screening of Children. Pediatrics 2013;131:620-622. 